\title{
Equivalência semântica da versão em português da Escala de Experiência de Quase-Morte
}

\author{
Fernanda Barcellos Serralta ${ }^{1}$ - Universidade Luterana do Brasil, Canoas, Brasil \\ Fernanda Cony - Universidade Luterana do Brasil, Canoas, Brasil \\ Zelia Cembranel - Universidade Luterana do Brasil, Canoas, Brasil \\ Bruce Greyson - University of Virginia Health System, Virginia, United States \\ Cláudia Maciel Szobot - Universidade Luterana do Brasil, Canoas, Brasil
}

\begin{abstract}
Resumo
$\mathrm{Na}$ literatura internacional, a experiência de quase-morte (EQM) é avaliada principalmente por meio do instrumento The Near-Death Experience Scale (NDE), elaborado por Bruce Greyson, em 1983. O objetivo do presente estudo é verificar a existência de equivalência semântica entre a versão original da escala (em inglês) e a versão traduzida ao português do Brasil, avaliando os significados geral e referencial. Após as diversas etapas de avaliação da equivalência semântica, a escala foi pré-testada em seis pacientes internados após alta de um Centro de Tratamento Intensivo (CTI) de um Hospital Universitário. Na avaliação realizada por dois psicólogos e dois psiquiatras, constatou-se existir equivalência semântica entre a versão final em português e o original. Na aplicação da escala na população-alvo, verificou-se boa compreensão dos itens. Portanto, a escala está adequada à cultura brasileira, podendo ser utilizada para pesquisas sobre Experiências de Quase-Morte neste contexto.

Palavras-chave: Experiência de Quase-Morte (EQM), Equivalência Semântica, Escala.
\end{abstract}

\section{Semantic equivalence of the Portuguese version of the Near-Death Experience Scale}

\begin{abstract}
In the international literature, near-death experiences are often measured by The Near-Death Experience Scale (NDE), developed by Bruce Greyson in 1983. The aim of this study is to verify the existence of the semantic equivalence between the original version (in English) and the translated version to Brazilian Portuguese, evaluating both the general and the referential meaning. The semantic evaluation was conducted according to the several steps. Thereafter, a pre-testing was performed in six post Intensive Care Unit patients of a University Hospital. The evaluations of two psychologists and two psychiatrists have found existence of semantic equivalence between the Brazilian and the original version of the scale. The pre-test applied on the target population proved the items were intelligible. Our findings suggest that the Brazilian version of the NDE might be used in the investigation of near-death experience in Brazilian culture.

Keywords: Near-Death Experience (NDE), Semantic Equivalence, Scale.
\end{abstract}

A Experiência de Quase-Morte (EQM) envolve um conjunto de experiências extraordinárias ou místicas relatadas por pessoas que estiveram no limiar da morte e que, todavia, conseguiram sobreviver. É comum em pacientes terminais, sobreviventes de morte clínica, e em situações em que haja momentos de perigo extremo, tais como infarto do miocárdio, choque anafilático, intoxicação, traumatismo, anestesia, tentativa de suicídio, hemorragia cerebral e afogamento (Fernandes, 1998; Greyson, 2003; van Lommel, van Wees, Meyers \& Elfferich, 2001).

Ainda que EQMs sejam descritas na literatura médica desde o século XIX (Greyson, 2007b), seus relatos têm se tornado mais frequentes

1 Endereço para correspondência:

Rua Cel. Corte Real 797/502 - Petrópolis - 90630-080Porto Alegre - RS

E-mail: psifer@terra.com.br provavelmente devido às taxas de sobrevivência resultantes das técnicas modernas de ressucitação (van Lommel \& cols., 2001). Estudos com sobreviventes de paradas cardíacas revelam que entre $10 \%$ e $18 \%$ relatam experiências subjetivas profundas consistentes com a noção de "experiência de quase-morte" (Greyson, 2007b; Parnia \& Fenwick, 2002).

Muito pouco se sabe acerca dos estados mentais no final da vida e obviamente não há como se investigar os estados de consciência diante da morte consumada. Desse modo, a investigação de tais fenômenos e experiências é potencialmente valiosa no estudo da consciência, seus mecanismos psicológicos e sua relação com o funcionamento cerebral (French, 2005; Greyson, 2007b; Parnia \& Fenwick, 2002; Parnia, Waller, Yeates \& Fenwick, 2001).

EQMs envolvem estados alterados de consciência e, embora possam lembrar estados 
psicopatológicos, envolvem sequelas diferentes e requerem uma abordagem terapêutica também diferenciada (Athappilly, Greyson \& Stevenson, 2006). Entretanto, como bem salientam Almeida e Lotufo Neto (2003), estados "paranormais", místicos e religiosos relacionados às experiências anômalas e estados alterados de consciência, embora comuns na população geral, são usualmente negligenciados pelos pesquisadores e estudiosos. Profissionais de saúde mental não são treinados para lidar com essas manifestações.

$O$ ato de considerar patológica uma experiência dissociativa particular é grandemente influenciado pela cultura (Lewis-Fernández, 1998), a qual pode influenciar a prática clínica de diversas formas: como instrumento explicativo, agente patogênico ou patoplástico, como fator diagnóstico e como elemento terapêutico e protetor (Alarcón, Westermeyer, Foulks \& Ruiz, 1999).

$\mathrm{Na}$ literatura internacional a EQM é avaliada principalmente através do instrumento The NearDeath Experience Scale (NDE) (Greyson, 1983a). Não há, entretanto, uma tradução e adaptação deste instrumento (ou de similar) ao português do Brasil, assim limitando pesquisas nesta área. O objetivo do presente estudo é a realização da adaptação transcultural da The Near-Death Experience scale para o português, especificamente no que se refere à avaliação de equivalência semântica, propondo uma versão final em língua portuguesa para uso corrente no Brasil.

\section{Experiência de Quase-Morte (EQM)}

O termo "Experiências de Quase-Morte", tradução de "Near-death Experiences", cunhado pelo psiquiatra americano dr. Raymond Moody Jr., surgiu com a publicação do seu livro $A$ vida depois da vida, em 1975. Moody Jr. (2004) descreve as experiências de 150 pessoas que viveram o fenômeno de quase-morte. Constatou que existem experiências comuns à maioria das pessoas que passaram pela EQM, tais como, dificuldade para descrever a experiência em palavras; ouvir o anúncio da sua própria morte; ouvir um zumbido desagradável ou música agradável nos ouvidos; ter um sentimento de paz e ausência de dor; ter uma experiência fora do corpo; sentir-se a viajar dentro de um túnel; ver "espíritos" ou pessoas, principalmente familiares já falecidos; ver uma revisão da própria vida; e sentir uma enorme relutância em voltar à vida. De acordo com Morse, Castillo, Venecia, Milstein e Tyler (1986), ainda que a maioria dos estudos encontrados na literatura sejam realizados com adultos, há indicativos que EQMs também ocorrem em crianças e adolescentes, de forma similiar, desencadeadas pelo processo de morrer ou pelos procedimentos de ressurreição.

Existem diversas hipóteses explicativas acerca da etiologia das EQMs, entre elas mecanismos biológicos, psicológicos e sociopsicológicos. A causa dessas experiências, entretanto, permanece desconhecida (French, 2005; Greyson, 2000). As teorias biológicas acentuam a relação da EQM com hipoxia cerebral, anoxia, hipercarbia, funcionamento de neurotransmissores e atividades anormais nos lobos temporais (French, 2005). Teorias psicológicas salientam que EQMs parecem constituir a expressão da dissociação como mecanismo de defesa diante de situações de perigo extremo (French, 2005; Greyson, 2000).

Tendo investigado 40 relatórios médicos de pacientes que relataram experiências não usuais (ver o próprio corpo de uma posição diferente no espaço, por exemplo) durante uma condição clínica, Stevenson, Cook e McClean-Rice (1989) encontraram que $45 \%$ das pessoas estiveram, de fato, em situações que ameaçaram seriamente a vida, enquanto $55 \%$ dos pacientes não tiveram nenhuma condição onde a vida estivesse em risco. Outro resultado sugestivo diz respeito ao percentual $(82,5 \%)$ dos sujeitos que acreditaram que, dentro da situação clínica, estivessem "mortos" ou próximos da morte. Tais resultados levaram os autores a afirmar que um dos fatores precipitadores das chamadas experiências de quase-morte pode ser a crença que o paciente tem de que está efetivamente morrendo, muito embora do ponto de vista clínico não o esteja. Essa discrepância entre uma avaliação subjetiva (crença do experienciador) e uma de natureza mais objetiva (avaliação clínica do médico) introduz uma variável complexa na compreensão da EQM. Greyson (1983b) constatou que EQMs precipitadas por eventos de quase-morte antecipados apresentavam menos elementos da dimensão cognitiva (tempo veloz, pensamentos velozes, visão retrospectiva, compreensão ampliada) quando comparadas com a EQM eliciada por eventos súbitos e inesperados de quase-morte, o que indica que fatores psicológicos podem influenciar o tipo de EQM. Outro estudo, realizado por Owens, Cook e Stevenson (1990) comparou os relatos de dois tipos de pacientes que passaram por EQMs: pacientes que estiveram, de fato, em situação de risco de vida, e pacientes que acreditavam ter estado perto da morte, mas que do 
ponto de vista clínico não estiveram. Embora os relatos sejam semelhantes na maioria dos aspectos, os pacientes que realmente estiveram próximos da morte (e, por conseguinte, presumidamente com maior alteração da função cerebral) paradoxalmente apresentaram mais relatos de percepção da luz e cognição aumentadas. Esses achados apontam para a necessidade de mais pesquisa no assunto.

Muitas características das EQM sugerem a presença de mecanismos dissociativos. Em um estudo que investigou a frequência e o tipo de dissociação entre pacientes que tiveram experiências de quase-morte e em pessoas que estiveram próximas da morte, mas não tiveram experiência de quase-morte, Greyson (2000, p. 463) concluiu que

EQMs não parecem ser um tipo patológico de dissociação on a manifestação de uma doença dissociativa; parecem ser experiências nãopatológicas que envolvem o mecanismo de dissociação como uma resposta normal a um trauma intolerável.

Conforme Moody Jr. (2004), de modo geral, nos primeiros passos dessa experiência é frequente a descrição de sentimentos de paz muito agradáveis. Embora as pessoas que tiveram uma EQM evitem falar sobre o evento pelo medo de serem consideradas desequilibradas mentalmente, costumam lembrar com exatidão cada detalhe do que lhes ocorreu e, ainda que não seja relatada, a experiência fica viva em suas mentes como um filme assistido há poucos minutos. No prefácio do livro de Moody, a dra. Elizabeth Kübler Ross dá seu depoimento sobre os importantes estudos iniciados por ele. Durante muitos anos, Kübler Ross assistiu e pesquisou diversos pacientes em estado terminal e teve a oportunidade de constatar que muitos deles passaram por experiências de quase-morte. Com base em suas observações, desenvolveu um projeto de estudo sobre a EQM em cegos, principalmente nos que não tiveram nenhum vislumbre de luz pelo prazo de no mínimo dez anos. Muitos desses pacientes conseguiram, durante uma EQM, ver cenas e descrever com riqueza de detalhes até mesmo como as pessoas estavam vestidas.

Uma EQM produz diversos efeitos psicológicos, entre os quais se destacam: a) redução ou extinção do medo da morte e maior gosto pela vida; b) conscientização da importância do amor; c) sensação de união com todas as coisas; d) valorização do conhecimento; e) maior responsabilidade pela própria vida; f) ampliação do vigor e da atividade mental e física; g) aparente rejuvenescimento; h) reavaliação das coisas materiais da vida; i) profundo senso de missão; j) mudança carismática na personalidade; l) desenvolvimento súbito ou gradual de aptidões; $\mathrm{m})$ prazer pelo conhecimento enciclopédico; $\mathrm{n}$ ) sentido de urgência e reavaliação de prioridades. Depois de uma EQM, os sobreviventes tendem a experimentar grandes mudanças comportamentais e de identidade pessoal: gostam mais de si mesmos, mostram uma apreciação maior da vida e maior preocupação e amor pelos outros, enquanto diminui seu interesse em status pessoal e posses materiais. A maioria dos sobreviventes também declara que vive depois com um sentido de finalidade espiritual ampliado e, em alguns casos, que procura um entendimento maior do significado essencial da vida (Ring, 1996).

As pesquisas revelam que na maioria das vezes as EQMs trazem efeitos positivos na vida das pessoas que as experienciam (Greyson, 2000, 2003; 2007a). Ao examinar os efeitos da EQM sobre a vida de 215 sujeitos, Noyes (1980) encontrou um padrão de mudanças positivas em termos de atitudes, valores, crenças e condutas, tais como redução do medo da morte, sensação de relativa invulnerabilidade, sentimento de importância especial ou destino, forte crença na continuidade da existência, sensação de preciosidade da vida, sentido de urgência e reavaliação de prioridades e atitude mais passiva (compreensiva) diante de eventos que escapam ao controle do sujeito. Há ainda evidências de que a EQM, ao promover significativas transformações nos valores das pessoas, diminui a ideação suicida (Greyson, 1983b; Greyson, 1993) e sintomas de estresse pós-traumático (Greyson, 2001) e aumenta a espiritualidade (Greyson, 2006). Estar próximo da morte está associado a maior sofrimento psicológico. Entretanto, passar por uma EQM mostra ser um fator associado à diminuição desse sofrimento (Greyson, 2003).

Fernandes (1998) afirma que os estudos sobre EQM estão centrados em pelo menos um dos seguintes aspectos: Descrição fenomenológica. São investigados os elementos comuns a uma EQM, estabelecendo-se uma verdadeira tipologia da experiência; Aspectos pragmáticos - efeitos sobre a conduta, sistema de crenças e valores, mudanças na personalidade; nova atitude diante da vida e da morte; ideação suicida; Aspectos etiológicos - tenta-se desenvolver uma explicação coerente para sua ocorrência; significativas divergências teóricas, conceptuais; Atitudes e conbecimento em relação à 
EQM - escalas, questionários, instrumentos de medida para avaliar o conhecimento e as atitudes de sujeitos em relação à EQM.

Há evidências de que a EQM pode apresentar algumas variações culturais. Em um estudo realizado no sul da Índia (Pasricha \& Stevenson, citados por Fernandes, 1998), foi encontrado que nenhum dos 16 indivíduos avaliados relatou ter ocorrido experiência fora do corpo durante a EQM. Esta e outras variações entre uma cultura e outra sugere que idéias acerca da vida após a morte podem exercer alguma influência no conteúdo de uma EQM. Também uma investigação realizada na China com sobreviventes de um grande terremoto ocorrido em 1976 sugere que os componentes e os tipos de EQM podem estar relacionados a fatores psicológicos, culturais e ao tipo de evento (Feng, 1982). Esses dados reforçam a importância da avaliação da EQM em diferentes culturas.

\section{Adaptação transcultural}

Conforme Hauck e cols. (2006), a avaliação semântica é um dos aspectos importantes na adaptação de instrumentos de pesquisa nas diferentes culturas, pois envolve questões culturais e de linguagem que podem comprometer a validade conceitual $e$ as propriedades psicométricas do instrumento. Várias etapas são necessárias para que ocorra a validação de instrumento.

Os estudos transculturais são norteados por duas diferentes perspectivas, representando o "paradigma emic-etic" (Brislin \& cols., 1973, citados por Jorge, 1998). A perspectiva emic se refere à avaliação de um fenômeno a partir da própria cultura onde o estudo é realizado, tendo como objetivo caracterizar a lógica interna dessa cultura e sua singularidade, pré-requisito para qualquer análise transcultural válida. Já a perspectiva etic deduz das especificidades locais categorias mais gerais do fenômeno, a fim de identificar e comparar fenômenos equivalentes em contextos culturais diferentes. Quando o instrumento de avaliação for desenvolvido na cultura onde será utilizado, seu uso vai necessitar de um processo de tradução e adaptação abrangente, com o objetivo de buscar uma equivalência cultural que não é alcançada apenas por técnicas de tradução e retrotradução. Os fatores linguísticos e socioculturais múltiplos devem ser considerados na determinação de equivalência transcultural (Jorge, 1998).
Segundo o modelo desenvolvido por Herdman, Fox-Rushby e Badia (1998), e aplicado em pesquisas nacionais por Reichenheim, Moraes e Hasselmann (2000); Reichenheim e Moraes (2003); Alves, Chor, Faerstein, Lopes e Werneck (2004); e Fiszman e cols. (2005), para que haja a equivalência transcultural de um instrumento, é preciso que este seja submetido à análise ordenada de seis subtipos de equivalências, quais sejam, conceitual, de itens, semântica, operacional, de mensuração e funcional.

Conforme Reichenheim e Moraes (2007), a avaliação da equivalência conceitual consiste na exploração do construto de interesse e dos pesos dados aos seus diferentes domínios constituintes no local (país, região, cidade) de origem e na população-alvo onde o instrumento será utilizado. A equivalência de itens requer a análise de uma comissão de especialistas (juízes ou peritos no assunto), uma revisão da literatura e o julgamento de membros da população-alvo. A equivalência operacional se refere a uma comparação entre os aspectos de utilização de um instrumento nas populações-alvo e fonte, de modo que a eficácia seja semelhante mesmo que os modus operandi não sejam os mesmos. A equivalência de mensuração se baseia na investigação das propriedades psicométricas do instrumento vertido. A equivalência funcional refere-se a quanto o instrumento mede na cultura-alvo e o que se propõe a medir na cultura de origem.

\section{Equivalência semântica}

De acordo com Reichenheim e Moraes (2007, p. 665-673),

avaliação da equivalência semântica envolve a capacidade de transferência de sentido dos conceitos contidos no instrumento original para a versão, propiciando um efeito nos respondentes semelhante nas duas culturas.

Herdman e cols. (1998) preconizam a utilização de um modo universalista de abordar a pesquisa transcultural, que pretende avaliar e respeitar as diferenças culturais. Essa abordagem implicaria a necessidade de estabelecer se os conceitos compreendidos em determinado instrumento existem e são interpretados similarmente nas duas culturas em questão e, se sim, em que grau são interpretados similarmente. Um dos aspectos a ser considerado na adaptação transcultural se refere à avaliação de equivalência entre o original e cada uma das retraduções sob a perspectiva do significado referencial dos termos constituintes e as idéias ou objetos do mundo a 
que uma ou várias palavras se referem. Outro aspecto diz respeito ao significado geral de cada item do instrumento, contrastando-se o original com o que foi captado na tradução para o idiomaalvo. Essa correspondência transcende a literalidade das palavras, levando em consideração também aspectos mais sutis, como o impacto que um termo tem no contexto cultural da populaçãoalvo. A apreciação é necessária porque a correspondência literal de um termo não implica que a mesma reação emocional ou afetiva seja evocada em diferentes culturas. Essa questão é particularmente relevante em instrumentos para a captação empírica de conceitos culturalmente construídos, pois uma palavra ou assertiva usada com uma determinada intenção no contexto de origem pode não produzir o mesmo efeito na população-alvo da nova versão.

\section{Método}

\section{Instrumento}

A Escala de Experiência de Quase-Morte (Near-Death Scale) foi elaborada por Greyson (1983a) com base em um conjunto de manifestações características de EQM. Os itens do instrumento contemplam quatro dimensões que compõem as EQMs: cognitiva (tempo veloz, pensamentos acelerados, visão retrospectiva, compreensão ampliada, por exemplo), afetiva (sentimento de paz, prazer, calma, unidade com o universo e outros), paranormal (cenas do futuro, separação mente-corpo) e transcendental (ver pessoas mortas, seres de luz). A escala preliminar, com 33 itens, foi aplicada numa amostra de 74 sujeitos que teriam supostamente experienciado fenômenos característicos de uma EQM. Os itens que não apresentaram correlação com o total da escala foram retirados e sua versão final contém 16 itens pontuados numa escala tipo Likert de 0 a 3. Um escore igual ou maior que 7 indica a presença de uma EQM. Os estudos originais realizados com a escala demonstraram que a mesma possui alta consistência interna (coeficiente alfa de 0,88), precisão entre duas metades (coeficiente alfa de 0,84), estabilidade temporal (coeficiente alfa de 0,92 ) e validade convergente e discriminante satisfatórias.

\section{Procedimentos}

A tradução do instrumento The Near-Death Experience Scale para o português foi realizada mediante consentimento do autor, por comunicação eletrônica, em junho de 2007. O processo utilizado foi baseado nas diretrizes gerais do método proposto por Herdman e cols. (1998), introduzido no Brasil por Reichenheim e cols. (2000). Foram realizadas oito etapas consecutivas, cada uma delas com diferentes participantes, como descrito a seguir.

A etapa 1 consistiu na elaboração de duas traduções independentes da versão original do instrumento (VO) em inglês para o português. Os participantes nessa etapa foram dois profissionais fluentes no idioma inglês, um graduado em Psicologia (T1) e outro em Serviço Social (T2). Nessa etapa, respeitou-se a equivalência operacional, que objetiva manter características originais, conferindo maior confiabilidade e validade do instrumento (Herdman \& cols., 1998), mantendo o mesmo número de itens (16), o mesmo enunciado e as mesmas três opções de respostas.

A etapa 2 consistiu na unificação das traduções T1 e T2, realizada pelos autores, gerando a versão 1 (V1).

A etapa 3 consistiu na retrotradução (R1) da V1 para o inglês, por um profissional bilíngue cuja língua nativa é o inglês.

A etapa 4 consistiu na apreciação formal de equivalência semântica por quatro profissionais: dois psicólogos e dois psiquiatras. Todos os participantes dessa etapa eram bilíngues; nenhum tinha conhecimento prévio do instrumento. Para o julgamento da equivalência semântica, avaliaram-se os significados geral e referencial dos termos e das expressões de cada um dos 16 itens que compõem a escala (Herdman \& cols., 1998; Reichenheim \& cols., 2000). Para avaliar o significado referencial, julgou-se a equivalência entre os itens do instrumento original (VO) e da retrotradução (R1) de forma contínua, com notas variando entre 0 e $100 \%$ em uma escala analógica visual.

Os significados gerais representam as ideias (conceitos) a que uma única palavra ou um conjunto de palavras aludem, levando em conta aspectos mais sutis que a correspondência literal. Assim, na equivalência semântica, leva-se em consideração não apenas o significado das palavras entre dois idiomas diferentes, mas também o efeito que os itens (perguntas) têm em culturas distintas. Para cada item, foi utilizado um formulário no qual a versão 1 (V1) e retrotradução (R1) eram apresentados e os profissionais julgavam a equivalência em quatro níveis: inalterado (IN), pouco alterado (PA), muito alterado (MA) e completamente alterado (CA).

A etapa 5 , realizada pelos autores, consistiu em ajustar os itens do instrumento tomando por 
base avaliações obtidas na etapa 4, gerando a versão 2 (V2).

A etapa 6 consistiu na aplicação da V2 em uma amostra de conveniência composta de 6 pacientes internados, após alta da CTI, num Hospital Universitário para verificar a compreensão dos itens. Todos os participantes eram adultos e assinaram o Termo de Consentimento Livre e Esclarecido previsto no protocolo do estudo. A idade do grupo variou entre 30 e 83 anos, sendo a idade média 61 anos. Um dos participantes possuía ensino fundamental completo, dois, ensino médio completo, um, ensino superior incompleto e os outros dois, ensino superior completo. Conforme indicação do médico responsável pelo CTI, todos haviam passado por grave risco de morte e se recuperado satisfatoriamente. Os motivos da internação foram variados, mas a parada cardíaca a mais prevalente, presente em três casos. Em virtude das condições clínicas dos pacientes, cinco responderam o instrumento de forma oral. $\mathrm{Na}$ ocasião, falas espontâneas dos pacientes foram registradas por escrito a fim de subsidiar dados complementares.

A etapa 7 consistiu na discussão dos itens que apresentaram problemas de entendimento pela população avaliada.

A etapa 8 consistiu na elaboração da retrotradução (R2) da V2 e no envio para o autor original, que aprovou a versão.

\section{Resultados}

De modo geral, as avaliações realizadas pelos juízes especialistas indicam uma boa equivalência semântica entre a $\mathrm{VO}$ e a V1. Em relação ao significado referencial, a equivalência variou de 54\% a 99\% nos 16 itens. Apenas 1 item (item $9-$ os seus sentidos estavam mais agucados que o normal?) obteve avaliação do significado referencial abaixo de $70 \%$. Assim, de modo geral, verificou-se que houve correspondência literal entre as duas versões. No que concerne ao significado geral, constata-se que em $70,4 \%$ das avaliações o significado foi considerado inalterado e em $20,3 \%$, pouco alterado. Apenas uma avaliação de 1 item (5 - você teve um sentimento de paz ou serenidade?) o considerou completamente alterado. Dos itens avaliados, $7,8 \%$ foram julgados com significado geral muito alterado, indicando a necessidade de ajustes na versão em português do instrumento. Esses ajustes, realizados na etapa 5, após a discussão entre os autores, estão descritos na Tabela 1.

Dos 16 itens, 11 não sofreram qualquer modificação a partir da primeira tradução e retradução. Porém, alguns ajustes foram feitos para melhor adequar as frases traduzidas para a língua portuguesa coloquial. $\mathrm{Na}$ equivalência do significado geral, os itens 1,2 e 9 foram julgados como "pouco alterados" por causa da palavra "normal". Esta foi substituída por "habitual", pois possui um só sentido, o que proporciona maior compreensão. Embora seja a tradução correta de "usual", ela pode não ser corretamente compreendida em nosso contexto cultural, devido ao sentido de "desvio" ou de "patológico" que possui.

Tabela 1 - Modificações realizadas na etapa 5 (continua)

\begin{tabular}{|c|c|c|}
\hline Versão original & Versão 1 & Versão 2 \\
\hline $\begin{array}{l}\text { 1. Did time seem to speed } \\
\text { up or slow down? } \\
0=\text { No } \\
1=\text { Time seemed to go } \\
\text { faster or slower than usual } \\
2=\text { Everything seemed to be } \\
\text { happening at once; or time } \\
\text { stopped or lost all meaning }\end{array}$ & $\begin{array}{l}\text { 1. O tempo pareceu ter acelerado } \\
\text { ou ter passado mais devagar? } \\
0=\text { Não } \\
1=\text { O tempo pareceu ter passado } \\
\text { mais rápido ou devagar do que o } \\
\text { normal } \\
2=\text { Tudo pareceu estar } \\
\text { acontecendo de uma só vez; ou o } \\
\text { tempo parou ou perdeu todo o seu } \\
\text { significado }\end{array}$ & $\begin{array}{l}\text { 1. O tempo pareceu ter acelerado } \\
\text { ou ter passado mais devagar? } \\
0=\text { Não } \\
1=\text { O tempo pareceu ter passado } \\
\text { mais rápido ou devagar do que o } \\
\text { habitual } \\
2=\text { Tudo pareceu estar } \\
\text { acontecendo de uma só vez; ou o } \\
\text { tempo parou ou perdeu todo o } \\
\text { seu significado }\end{array}$ \\
\hline $\begin{array}{l}\text { 2. Were your thoughts } \\
\text { speeded up? } \\
0=\text { No } \\
1=\text { Faster than usual } \\
2=\text { Incredibly fast }\end{array}$ & $\begin{array}{l}\text { 2. Seus pensamentos ficaram mais } \\
\text { rápidos? } \\
0=\text { Não } \\
1=\text { Mais rápidos do que o normal } \\
2=\text { Incrivelmente rápidos }\end{array}$ & $\begin{array}{l}\text { 2. Seus pensamentos ficaram mais } \\
\text { rápidos? } \\
0=\text { Não } \\
1=\text { Mais rápidos do que o } \\
\text { habitual } \\
2=\text { Incrivelmente rápidos }\end{array}$ \\
\hline
\end{tabular}


Tabela 1 - Modificações realizadas na etapa 5 (conclusão)

Versão original

5. Did you have a feeling of

peace or pleasantness?

$0=$ No

$1=$ Relief or calmness

$2=$ Incredible peace or pleasantness

8. Did you see, or feel surrounded by, a brilliant light?

$0=$ No

$1=$ An unusually bright light

$2=$ A light clearly of

mystical or other-worldly origin

9. Were your senses more vivid than usual?

$0=$ No

$1=$ More vivid than usual

$2=$ Incredibly more vivid

Versão 1

5. Você teve um sentimento de paz ou de serenidade?

$0=\mathrm{Não}$

$1=$ Alívio ou calma

$2=$ Incrível paz ou serenidade
Versão 2

5. Você teve um sentimento de

paz ou de bem-estar?

$0=$ Não

$1=$ Alívio ou calma

$2=$ Incrível paz e bem-estar
8. Você viu ou se sentiu rodeado(a) por uma luz brilhante?

$0=$ Não

$1=$ Uma luz com um brilho fora

do normal

$2=$ Uma luz de origem claramente

mística ou de outro mundo
8. Você viu ou se sentiu

rodeado(a) por uma luz brilhante?

$0=$ Não

$1=$ Uma luz com um brilho

incomum.

$2=$ Uma luz de origem

claramente mística ou de outro mundo

9. Os seus sentidos estavam mais aguçados que o normal?

$0=$ Não

$1=$ Mais aguçados que o normal

$2=$ Incrivelmente mais aguçados
9. Os seus sentidos estavam mais aguçados que o habitual?

$0=$ Não

1 = Mais aguçados que o habitual

$2=$ Incrivelmente mais aguçados
No item cinco a palavra "bem-estar" (em inglês, pleasantness) nos pareceu mais adequada do que "serenidade", já que dois avaliadores consideraram a questão como muito alterada e completamente alterada. No item oito, o termo "unusually" (fora do normal) foi modificado por "incomum" por ser mais apropriado para a nossa realidade e não possuir a conotação de "patológico".

A palavra "vivid" utilizada na versão original foi retraduzida por "sharper" (vigarista, trapaceiro). $\mathrm{Na}$ avaliação do significado geral a mesma foi considerada por dois avaliadores como "pouco alterada". Após discussão, concluiu-se que esse fato ocorreu por problemas de retrotradução, por isso, optamos pela palavra "aguçados".

Após as alterações realizadas no instrumento que gerou a V2, essa versão foi administrada nos 6 pacientes que tiveram alta do
Centro de Tratamento Intensivo (CTI), para a avaliação da compreensão dos itens do instrumento. Os escores na escala variaram entre 2 e 16 pontos, tendo dois respondentes ultrapassado o ponto de corte ( 7 pontos) que indica a presença de uma EQM verdadeira. Os itens da escala foram avaliados como de fácil compreensão por todos os sujeitos, exceto a sra. D, que apresentou dificuldade em compreender o item 9 (Os seus sentidos estavam mais agucados que o habitual?). Quando inquirida, justificou não ter compreendido plenamente o significado da palavra "sentidos". Em praticamente todos os itens os pacientes fizeram comentários adicionais que foram registrados pelas pesquisadoras.

Após discussão entre os autores, concluiuse não ser necessário nenhum ajuste adicional na versão 2, a qual foi novamente retrotraduzida e enviada para o autor do original, que a aprovou. A Tabela 2 apresenta a escala em sua versão final.

Tabela 2 - Escala de Experiência de Quase-Morte - EQM (continua)

Por favor, circule um número $(0,1$, ou 2$)$ por questão para indicar a resposta que mais se aproxima da sua vivência durante sua experiência de quase morte.

1. O tempo pareceu ter acelerado ou ter passado mais devagar?

$0=$ Não.

$1=$ O tempo pareceu ter passado mais rápido ou devagar do que o habitual.

2 = Tudo pareceu estar acontecendo de uma só vez; ou o tempo parou ou perdeu todo o seu significado.

2. Seus pensamentos ficaram mais rápidos? 
Tabela 2 - Escala de Experiência de Quase-Morte - EQM (continua)

$0=$ Não.

1 = Mais rápidos do que o habitual.

2 = Incrivelmente rápidos.

3. Cenas do passado retornaram à sua mente?

$0=$ Não.

$1=$ Eu lembrei de muitos acontecimentos passados.

2 = Meu passado passou como um "filme" diante de mim, fora do meu controle.

4. Subitamente você pareceu compreender tudo?

$0=$ Não.

$1=$ Tudo a meu respeito ou a respeito dos outros.

$2=$ Tudo sobre o universo.

5. Você teve um sentimento de paz ou de bem-estar?

$0=$ Não.

$1=$ Alívio ou calma.

$2=$ Incrível paz e bem-estar.

6. Você teve um sentimento de alegria?

$0=$ Não.

$1=$ Felicidade.

$2=$ Incrível alegria.

7. Você teve uma sensação de harmonia ou de unidade com o universo?

$0=$ Não.

$1=$ Eu não me senti mais em conflito com a natureza.

$2=$ Eu me senti um só ou unido com o mundo.

8. Você viu ou se sentiu rodeado(a) por uma luz brilhante?

$0=$ Não.

$1=$ Uma luz com um brilho incomum.

$2=$ Uma luz de origem claramente mística ou de outro mundo.

9. Os seus sentidos estavam mais aguçados que o habitual?

$0=$ Não.

1 = Mais aguçados que o habitual.

$2=$ Incrivelmente mais aguçados.

10. Você pareceu estar consciente de coisas que aconteciam, em outros lugares, como se fosse percepção extrassensorial?

$0=$ Não.

$1=$ Sim, mas os fatos não foram verificados.

$2=$ Sim, e os fatos foram verificados.

11. Cenas do futuro apareceram para você?

$0=$ Não.

$1=$ Cenas do meu futuro pessoal.

$2=$ Cenas do futuro do mundo.

12. Você se sentiu separado(a) do seu corpo?

$0=$ Não.

$1=$ Eu perdi a consciência do meu corpo.

$2=$ Eu claramente deixei meu corpo e existia fora dele.

13. Você pareceu ter entrado num outro mundo, sobrenatural?

$0=$ Não.

$1=$ Um lugar desconhecido e estranho.

$2=\mathrm{Um}$ lugar claramente místico ou sobrenatural.

14. Você pareceu encontrar um ser ou presença mística? Ou escutar uma voz não identificável?

$0=$ Não.

$1=$ Eu escutei uma voz que não pude identificar.

$2=$ Eu encontrei um ser definitivo ou uma voz de origem claramente mística ou sobrenatural.

15. Você viu mortos ou espíritos religiosos? 
Tabela 2 - Escala de Experiência de Quase-Morte - EQM (conclusão)

$0=$ Não.

$1=$ Eu senti a presença deles.

$2=$ Eu realmente os vi.

16. Você chegou a uma fronteira ou ponto sem retorno?

$0=$ Não.

1 = Eu cheguei a uma decisão consciente de "retornar" à vida.

2 = Eu cheguei a uma barreira que não me foi permitido atravessar; ou fui "mandado(a) de volta" contra a minha vontade.

\section{Discussão}

O termo Experiência de Quase-Morte (EQM) para designar um fenômeno bastante comum no cotidiano de profissionais da saúde que trabalham com pacientes que se encontram gravemente enfermos ou no limiar da morte. Tal como afirma Greyson (2007b), a investigação científica da EQM é relevante aos profissionais de saúde por diversos motivos: porque afeta profundamente os valores, as crenças e as atitudes dos pacientes que a vivenciaram, porque pode ser confundida com estamos psicopatológicos e porque possui o potencial para ampliar a nossa compreensão acerca do funcionamento da consciência da interação cérebro-mente.

A necessidade de um instrumento válido e confiável para avaliar EQM em nosso meio é de grande importância, uma vez que inexistem escalas ou questionários adaptados para o português que contemplem esse tipo de fenômeno, que é bastante prevalente entre a população que esteve em situação clínica grave ou próxima da morte, em diversos países e culturas (Greyson, 2003; van Lommel \& cols., 2001, Pasricha, 1993).

Neste trabalho, relatamos o processo de elaboração da versão em português da Escala de Experiência de Quase-Morte - The Near-Death Scale (Greyson, 1983a) e, especificamente, a avaliação da sua equivalência semântica com a versão original.

A tradução de uma escala requer cuidados linguísticos, uma vez que termos podem ter diferentes abrangências e especificidades inerentes a cada idioma. Além disso, a validação semântica é necessária para a certificação de que o instrumento é compreensível a todos os membros da população à qual se destina (Pasquali, 1998). Ao realizar uma tradução existem diferentes passos que devem ser seguidos para garantir que se obtenha, além da literalidade da tradução (significado referencial), a mesma conotação e impacto na população-alvo (significado geral) (Reichenheim \& Moraes, 2007).
De modo geral, os resultados demonstram que a versão em português da Escala de Experiências de Quase-Morte apresentou um nível bastante satisfatório de equivalência semântica entre a versão em português e o original em inglês. Após os ajustes realizados a escala foi administrada a 6 pacientes com passagem recente pela CTI, que avaliaram de forma satisfatória a inteligibilidade dos itens. A versão respondida por esses pacientes foi aprovada pelo autor do instrumento original, sendo assim considerada equivalente à versão em inglês.

A aplicação do instrumento na população-alvo foi bastante proveitosa, não somente por ter permitido a verificação da compreensão dos itens, mas também pelos dados complementares que foram obtidos por meio das verbalizações espontâneas. Todos os pacientes avaliados relataram, em algum grau, experiências compatíveis com a noção de EQM, sendo que 2 apresentaram pontuação maior do que 7 no instrumento, o que, conforme o ponto de corte original, configura uma EQM verdadeira. Durante a aplicação da escala observou-se que os pacientes demonstravam necessidade de relatar os fatos ocorridos de maneira detalhada. Esse é um dado interessante, visto que pessoas que tiveram uma EQM costumam não falar sobre o evento, com medo de serem consideradas desequilibradas mentalmente. Ao perceber que os pesquisadores não viam esta experiência como algo anormal, sentiam-se livres para relatá-la.

Os pacientes avaliados descreveram ver pessoas, principalmente familiares falecidos, uma das características da EQM encontrada por Moody Jr.(2004) e que é comum a norte-americanos e indianos (Paricha, 1993). D relata: "Vi minha mãe e fiquei com medo, pois não esperava vê-la" (sic). Também o paciente B relatou: "Vi cadáveres por toda a parte" (sic).

Os comentários feitos por alguns pacientes nas questões 5, 6 e 16 indicam que a EQM pode eliciar sentimentos desagradáveis e desejo de retornar a vida. O "sentir uma grande relutância 
de voltar à vida" (questão 16) não foi observado no relato da população-alvo. Ao contrário disso, alguns disseram sentir muita vontade de voltar a vida, de não morrer. "Eu pensava: não posso morrer agora, tenho muita coisa pra fazer, quero ter filhos" (sic, Paciente A). "Senti que não ia resistir, mas não queria morrer" (sic, Paciente D). "Voltei porque estavam precisando de mim" (sic, Paciente C). Nas respostas das questões 5 e 6 , que fazem menção a sentimentos de paz, bem-estar e alegria, quatro entrevistados relataram sentimentos contrários, como "Tive sentimento de Pavor" (sic, Paciente A). "Fiquei angustiado" (sic, Paciente B). "Me senti sozinha" (sic, Paciente C). "Tive medo" (sic, Paciente E). Conforme Greyson e Bush (1992), esse tipo de sentimento negativo é bem menos frequente que os positivos e seu impacto na vida futura dos sujeitos, ainda desconhecido. Se tais manifestações são características distintas da população brasileira ou não, somente estudos subsequentes poderão confirmar.

Ring (1996) afirma que a maioria das pessoas que passaram por EQM apresenta consideráveis mudanças de crenças, valores, atitudes e comportamentos. Isso se constatou em dois pacientes avaliados, que relataram ter passado por mudanças, como "ser mais otimista, pensar positivo, valorizar a vida, cuidar mais de mim e não tanto dos outros como costumava fazer até então" (sic, Paciente A). "Dei mais valor à família. Fiquei mais sensivel para as coisas que me rodeiam" (sic, Paciente E).

No presente estudo, adotamos as 8 etapas do processo de adaptação e avaliação de equivalência semântica, que são parte do processo mais amplo de adaptação transcultural (Herdman \& cols., 1998). Conforme descrevem Reichenhem e Moraes (2007), o modelo universalista de adaptação transcultural supõe ainda a equivalência conceitual, dos itens, operacional, de mensuração e funcional. A versão em português da Escala de Experiência de Quase-Morte satisfez os critérios de equivalência semântica. Manteve-se o formato $\mathrm{da}$ escala original em todos os itens do instrumento. Portanto, considera-se que exista equivalência operacional. A fim de concluir o processo de adaptação à cultura brasileira, além de uma discussão com especialistas e população-alvo para examinar a equivalência conceitual e de itens, é necessário que se realizem estudos empíricos para verificar as propriedades psicométricas do instrumento. Em especial, recomenda-se a validação de construto, visto não existir um outro instrumento de referência (padrão-ouro) para exame da EQM em nosso meio.

\section{Referências}

Alarcón, R. D., Westermeyer, J., Foulks, E. F. \& Ruiz, P. (1999). Clinical relevance of contemporary cultural psychiatry. Journal of Nervous and Mental Disease, 187, 465-471.

Almeida, A. M. \& Neto, L. F. (2003). Diretrizes metodológicas para investigar estados alterados de consciência e experiências anômalas. Revista de Psiquiatria Clinica, 30(1), $21-28$.

Alves, M. G. M., Chor, D., Faerstein, E., Lopes, C. S. \& Werneck, G. L. (2004). Versão resumida da "Job Stress Scale": adaptação para o português. Revista de Saúde Pública, 38, 164171.

Athappilly G. K., Greyson B. \& Stevenson I. (2006). Do prevailing societal models influence reports of near-death experiences? a comparison of accounts reported before and after 1975. Journal of Nervous and Mental Disease, 194(3), 218-222.

Fernandes, H. (1998). Experiências de quase-morte: uma questão científica? Dissertação de Mestrado. Universidade Federal do Rio de Janeiro, Rio de Janeiro.

Fiszman, A., Marques, C., Berger, W., Volchan, E., Oliveira, L. A. S., Coutinho, E. S. F., Mendlowicz, M. \& Figueira, I. (2005). Adaptação transcultural para o português do instrumento Peritraumatic Dissociative Experiences Questionnaire, Versão Autoaplicativa. Revista de Psiquiatria do Rio Grande do Sul, 27(2), 151-158.

French, C. C. (2005). Near-death experiences in cardiac arrest survivors. Progress in Brain Research, 150, 351-367.

Greyson, B. (1983a). The Near-Death Experience Scale: construction, reliability and validity. Journal of Nervous and Mental Disease, 171(6), 369-375.

Greyson, B. (1983b). Near-death experiences and personal values. American Journal of Psychiatry, 140(5), 618-620.

Greyson B. (1993). Near-death experiences and antisuicidal attitudes. Omega, 26, 81-89.

Greyson, B. (2000). Dissociation in people who have near-death experiences: out of their bodies or out of their minds? The Lancet, 355, 460-463. 
Greyson, B. (2001). Posttraumatic stress symptoms following near-death experiences. American Journal of Orthopsychiatry, 71(3), 368-373.

Greyson, B. (2003). Near-death experiences in a psychiatric outpatient clinic population. Psychiatric Services, 54(12), 1649-1651.

Greyson, B. (2006). Near-death experiences and spirituality. Zygon, 41(2), 393-414.

Greyson, B. (2007a). Consistency of near-death experience accounts over two decades: are reports embellished over time? Resuscitation, 73, 407-411.

Greyson, B. (2007b). Experiências de quase-morte: implicações clínicas. Revista de Psiquiatria Clínica, 34(1), 116-125.

Greyson, B. \& Bush, N. E. (1992). Distressing near-death experiences. Psychiatry, 55(1), $95-$ 110.

Hauck, S., Schestatsky, S., Terra, L., Knijnik, L., Sanchez, P. \& Ceitlin, L. H. F. (2006). Adaptação transcultural para o português brasileiro do Parental Bonding Instrument. Revista de Psiquiatria do Rio Grande do Sul, 28(2), 51-58.

Herdman, M., Fox-Rushby, J. \& Badia, X. (1998). A model of equivalence in the cultural adaptation of HRQoL instruments: the universalist approach. Quality of Life Research Journal, 7(4), 323-335.

Jorge, M. R. (1998). Adaptação transcultural de instrumentos de pesquisa em saúde mental. Revista de Psiquiatria Clinica, 25(5), 233-239.

Lewis-Fernández, R. (1998). A cultural critique of the DSM-IV dissociative disorders section. Transcultural Psychiatry, 35, 387-400.

Moody Jr., R A. (2004). Vida após a vida. São Paulo: Butterfly. (Original publicado em 1975).

Morse, M., Castillo, P., Venecia, D., Milstein, J. \& Tyler, D. C. (1986). Childhood near-death experiences. American Journal of Desease of Children, 140(11), 1110-1114.

Noyes, R. (1980). Attitude change following neardeath experience. Psychiatry, 43, 234-242.

Owens, J. E., Cook, E. W. \& Stevenson, I. (1990). Features of "near-death experience" in relation to whether or not patients were near death. The Lancet, 336(8724), 1175-1177.
Parnia, S., Waller, D. G., Yeats, R. \& Fenwick, P. (2001). A qualitative and quantitative study of the incidence, features and etiology of near death experiences in cardiac arrest survivors. Resuscitation, 48(2), 149-156.

Parnia, S. \& Fenwick, P. (2002). Near death experiences in cardiac arrest: visions of a dying brain or visions of a new science of consciousness. Resuscitation. 52(1), 5-11.

Pasquali, L. (1998). Princípios de elaboração de escalas psicológicas. Revista de Psiquiatria Clínica, 25(5), 206-213.

Pasricha, S. (1993). A systematic survey of neardeath experiences in South India. Journal of Scientific Exploration, 7(2), 161-171.

Reichenheim, M. E. \& Moraes, C. L. (2003). Adaptação transcultural do instrumento "Parent-Child Conflict Tactics Scales (CTSPC)" utilizado para identificar a violência contra a criança. Cadernos de Saúde Pública, 19(6), 701-712.

Reichenheim, M. E. \& Moraes, C. L. (2007). Operacionalização de adaptação transcultural de instrumentos de aferição usados em epidemiologia. Revista de Saúde Pública, 41(4), 665-673.

Reichenheim, M. E., Moraes, C. L. \& Hasselmann, M. H. (2000). Equivalência semântica da versão em português do instrumento Abuse Assessment Screen: para rastrear a violência contra a mulher grávida. Revista de Saúde Pública, 34(6), 610-616.

Ring, K. (1996). Rumo ao ponto omega. Rio de Janeiro: Rocco.

Stevenson, I., Cook, E. W. \& McClean-Rice, N. (1989). Are persons reporting "near-death experiences" really near death? A study of medical records. Omega, 20(1), 45-54.

Van Lommel, P., van Wees, R., Meyers, V. \& Elfferich, I. (2001). Near-death experience in survivors of cardiac arrest: a prospective study in the Netherlands. The Lancet, 358(9298), 2039-2045.

Recebido em agosto de 2008 Reformulado em julho de 2009 Aprovado em novembro de 2009 
Sobre os autores:

Fernanda Barcellos Serralta é mestre em Psicologia Clínica (PUCRS), doutoranda em Psiquiatria (UFRGS), docente do curso de Psicologia da ULBRA, em Canoas - RS.

Fernanda Cony é acadêmica do curso de Psicologia da ULBRA, em Canoas - RS.

Zelia Cembranel é psicóloga.

Bruce Greyson é Medical Doctor da Division of Perceptual Studies do Department of Psychiatry \& Neurobehavioral Sciences da University of Virginia Health System na Virginia - USA.

Cláudia Maciel Szobot é doutora em Psiquiatria (UFRGS), docente dos cursos de Psicologia e Medicina da ULBRA, em Canoas - RS. 